\title{
Auditing access to outpatient rehabilitation services for children with traumatic brain injury and public insurance in Washington State
}

\author{
Molly M. Fuentes, MD, MS ${ }^{1,2,3}$, Leah Thompson, BA ${ }^{2}$, D. Alex Quistberg, PhD $^{3}$, Wren L. \\ Haaland, MPH ${ }^{2}$, Karin Rhodes, MD, MS $^{4}$, Deborah Kartin, PhD, PT ${ }^{1}$, Cheryl Kerfeld, PhD, \\ $\mathbf{P T}^{1}$, Susan Apkon, $\mathbf{M D}^{1}$, Ali Rowhani-Rahbar, MD, PhD $^{3,5}$, and Frederick P. Rivara, MD, \\ MPH $^{2,3,5,6}$ \\ ${ }^{1}$ University of Washington School of Medicine, Department of Rehabilitation Medicine, Seattle \\ WA \\ 2 Seattle Children's Research Institute, Seattle WA \\ ${ }^{3}$ Harborview Injury Prevention and Research Center, Seattle WA \\ ${ }^{4}$ Hofstra Northwell School of Medicine, Office of Population Health, Great Neck, NY \\ ${ }^{5}$ University of Washington School of Public Health, Department of Epidemiology, Seattle WA \\ 6 University of Washington School of Medicine, Department of Pediatrics, Seattle WA
}

\section{Abstract \\ Objective-Identify insurance-based disparities in access to outpatient pediatric neurorehabilitation services.}

Design-Audit study, with paired calls where callers posed as a mother seeking services for a
simulated child with history of severe traumatic brain injury (TBI) and public or private insurance.

Setting-Outpatient rehabilitation clinics in Washington State

Participants-195 physical therapy clinics (PTc), 109 occupational therapy clinics (OTc), 102 speech therapy clinics (STc) and 11 rehabilitation medicine clinics

Interventions-None

Corresponding Author: Molly Fuentes, Seattle Children's Research Institute, PO Box 5371, M/S CW8, Seattle WA 98145-5005. Telephone (206) 884-8203. molly.fuentes@ seattlechildrens.org.

Publisher's Disclaimer: This is a PDF file of an unedited manuscript that has been accepted for publication. As a service to our customers we are providing this early version of the manuscript. The manuscript will undergo copyediting, typesetting, and review of the resulting proof before it is published in its final citable form. Please note that during the production process errors may be discovered which could affect the content, and all legal disclaimers that apply to the journal pertain.

Acknowledgement of Presentation: This material was presented in poster format at the American Congress of Rehabilitation Medicine Annual Meeting in Chicago, Illinois on November 3, 2016.

Conflicts of Interest: None of the authors have financial conflict of interest to report.

Suppliers

\footnotetext{
a. $\quad$ Esri (ArcGIS Desktop 10.4), 380 New York Street, Redlands, CA 92373-8100

b. $\quad$ StataCorp LP (Stata 12), 4905 Lakeway Drive, College Station, TX 77845-4512
} 
Main outcome measures-Acceptance of public insurance, business days until the next available appointment.

Results-Therapy clinics were more likely to accept private versus public insurance (relative risk (RR) for PTc 1.33 (95\% confidence interval (CI) 1.22-1.44), OTc 1.40 (95\% CI 1.24-1.57), and STc 1.42 (95\% CI 1.25-1.62), with no significant difference for rehabilitation medicine (RR 1.10, 95\% CI 0.90-1.34). The difference in median wait time between clinics that accepted public versus only private insurance was 4 business days for PTc and 15 days for STc (p s.001) but not significantly different for OTc or rehabilitation medicine. When adjusting for urban and multidisciplinary clinic status, the wait at clinics accepting public insurance was $59 \%$ longer for PT (95\% CI 39-81\%), $18 \%$ longer for OT (95\% CI 7-30\%) and 107\% longer for ST (95\% CI $87-130 \%$ ) than at clinics accepting only private insurance. Distance to clinics varied by discipline and area within the state.

Conclusion-Therapy clinics were less likely to accept public versus private insurance. Therapy clinics accepting public insurance had longer wait times than clinics that accepted only private insurance. Rehabilitation professionals should attempt to implement policy and practice changes to promote equitable access to care.

\section{Keywords}

Rehabilitation; Children with Disabilities; Health Services for Persons with Disabilities; Health Equity; Health Services Accessibility

Traumatic brain injury (TBI) is a leading cause of acquired pediatric disability. ${ }^{1}$ A study of children hospitalized for TBI found 70\% reported cognitive dysfunction and 56\% physical dysfunction 12 months after injury. ${ }^{2}$ Nationally, $34 \%$ of children hospitalized for TBI have publicly funded insurance, ${ }^{3}$ and children with public insurance were more likely than those with private insurance to have unmet rehabilitation needs one year post-injury. ${ }^{2}$

A systematic review found children with public insurance (Medicaid or Children's Health Insurance Program (CHIP)), are less likely to receive specialty medical care and have difficulty finding physicians that accept public insurance. ${ }^{4}$ In one study, pediatric specialty appointments were denied to $66 \%$ of callers with public insurance versus only $11 \%$ with private (employer-based or privately purchased) insurance, and children with public insurance waited 22 days longer for an appointment than children with private insurance. ${ }^{5}$ Other studies also found access disparities based on insurance type. ${ }^{6-8}$

Insurance-based disparities around outpatient neurorehabilitation services have been demonstrated. Claims analysis showed adults with TBI and Medicaid insurance were less likely than those with private insurance to receive outpatient rehabilitation services. ${ }^{9}$ A study of pediatric TBI services in Washington State found most providers were located in urban areas and $70 \%$ of physical therapy/occupational therapy clinics and $80 \%$ of speech therapy/ cognitive therapy clinics self-reported acceptance of public insurance. ${ }^{10}$ The relationship between insurance type and wait times for outpatient rehabilitation in the United States has not been examined. 
This study used audit methodology to define insurance-based disparities in access to outpatient pediatric neurorehabilitation services, with access measured by acceptance of insurance plan and wait time to the next available appointment. Exploratory aims based on anecdotal experience assessed the relationship between insurance type, access, the number of therapy disciplines provided by the clinic, and the urban status of the clinic. Another exploratory aim evaluated insurance-based variation in the distance traveled to services.

The study team hypothesized that compared to private insurance, public insurance would be accepted less frequently by outpatient rehabilitation clinics and would be associated with longer wait times for services. Exploratory hypotheses were that multidisciplinary and nonurban status would be associated with higher rates of public insurance acceptance but longer wait times, and that callers with public insurance would travel further to access services.

\section{Methods}

\section{Study design}

This was an audit study where researchers posed as mothers and made scripted, paired calls to therapy and rehabilitation medicine clinics to determine: if the clinic provided services for a simulated child with sequelae from a TBI; if the clinic accepted the caller's insurance; and the number of business days until the next available appointment. The calls occurred between November 2, 2015 and March 17, 2016, with paired calls separated by three to five weeks. This study was conducted in Washington, a state where $41 \%$ of children are publicly insured, ${ }^{11}$ most by Medicaid managed care plans. In Washington State, children with family incomes up to $210 \%$ of the federal poverty level are eligible for Medicaid and children with family incomes up to $312 \%$ of the federal poverty level qualify for Washington's separate CHIP (5.5\% of children using public insurance in the state). ${ }^{12,13}$

\section{Clinical scenario and protocol}

Each clinic was called twice using a script in which the caller had moved and needed to establish rehabilitation care for her simulated child with the following sequelae of TBI: balance difficulties, diminished use of the dominant arm, and cognitive-communication impairments. The paired calls varied by insurance status (private insurance or Medicaidmanaged care plan, hereafter referred to as public insurance) and child's age, gender and time since injury (eight-year-old girl nine months post-TBI or nine-year-old boy ten months post-TBI). The time frames represent a phase of injury beyond acute inpatient rehabilitation but when functional change is expected. The order of these characteristics was assigned using an Excel random number generator. ${ }^{14}$ If not asked about insurance, the caller confirmed the clinic accepted their insurance after obtaining the date of the next available appointment. The public insurance plan with the largest market share was used in this study, with the exception of calls to clinics in one county where the primary plan was not utilized. After completing paired calls, a team member called the clinics which did not accept the public insurance plan in the study to confirm they did not accept public insurance. No appointments were scheduled and calls were ended as soon as the outcome information was obtained. Responses to possible scheduling barriers were included in the scripts, which can be found in the supplementary materials. 


\section{Sampling methods}

The sample of therapy clinics potentially offering pediatric neurorehabilitation services was generated from (1) marketing lists of licensed physical therapists, occupational therapists, and speech therapists; (2) lists of community therapy clinics and county-specific resources for children with special healthcare needs maintained by the state's tertiary children's hospital; (3) a pediatric resource list from the Brain Injury Alliance of Washington; and (4) a Google Maps ${ }^{15}$-based search using the terms "pediatric [physical/occupational/speech] therapy". Clinics identified by the children's hospital or Brain Injury Alliance and those with websites listing neurological or pediatric services were included. Therapy clinics were excluded if they provided only home health or specialized treatments (e.g. hand therapy), served only children under 3-years-old, focused on musculoskeletal or sports therapy, or did not accept any insurance.

The sample of rehabilitation medicine clinics was generated from publicly-available databases from the American Board of Physical Medicine and Rehabilitation, the American Academy of Physical Medicine and Rehabilitation, and major healthcare systems in Washington State. The rehabilitation medicine sample included those providing pediatric, neurological, or general outpatient rehabilitation care; clinics focused on musculoskeletal rehabilitation or electrodiagnostic testing were excluded.

\section{Outcomes and covariates}

This study's primary outcomes were the clinic's acceptance of public insurance and the number of business days until the next available appointment. Secondary outcomes included the distance from each Washington State census block group (CBG) center point to the five nearest clinics accepting any insurance type (since those with private insurance can access clinics accepting both insurance types) and the distance to the five nearest clinics accepting public insurance. Urban and multidisciplinary statuses of the clinic were covariates. Urban status was derived from the county-level Urban Influence Code $^{16}$ and classified as being in a large metropolitan (greater than one million people) or small/non-metropolitan area. Clinics were classified as single discipline if they offered only one discipline or multidisciplinary if two or more therapy services were available at the facility.

\section{Study oversight}

The University of Washington Human Subjects Division determined this study was not human subjects research, as clinics, not people, were studied. The study team independently obtained a research bioethics consultation from the University of Washington Institute of Translational Health Sciences, which recommended clinics be sent a letter indicating this study was taking place. Two clinics responded that they did not serve children with TBI and were removed from the sample.

\section{Statistical analysis}

Descriptive statistics characterized the population of clinics and insurance acceptance patterns. Generalized linear models (GLM), using Poisson family and log link, with a random effect for clinic to account for repeated measures from paired calls and robust variance estimations compared the proportion of clinics accepting private versus public 
insurance. This analysis was then stratified by multidisciplinary and urban status. Because baseline risk for insurance acceptance between callers with public and private insurance was hypothesized to be different, Poisson-based GLM were thought to be superior to other forms of regression. ${ }^{17}$

Median wait time for callers with public insurance at clinics accepting public insurance was compared to the wait time at clinics accepting only private insurance using the Wilcoxon rank-sum on median difference test. For clinics accepting both insurance types, the intraclinic difference in number of business days until the next available appointment with public versus private insurance was calculated. A multivariable GLM, using Poisson family and log link with robust variance for wait times in business days until an appointment, assessed the influence of insurance type acceptance and clinic's multidisciplinary and urban status on wait time for each discipline. In this model, the wait time at clinics accepting public insurance was the wait time for callers with public insurance. The estimates from this model are interpreted as count ratios describing the factor by which the count of business days is greater at clinics accepting public insurance compared to those accepting only private insurance. Because only one rehabilitation medicine clinic did not accept public insurance and one STc did not accept private insurance, these clinics were excluded from analyses comparing wait times by types of insurance accepted.

\section{Geospatial analysis}

Because families likely utilize geographically accessible services, geospatial analysis was conducted to assess the average driving distance and wait time by CBG. Clinics were geocoded by latitude and longitude from street addresses using GPS Visualizer via the Bing Maps API. ${ }^{18,19}$ Center points from the centroid of the polygon of each CBG in Washington were generated. Driving distance (miles) from a CBG center point to a clinic was determined by creating a road network dataset with the US Census and Washington public roads geodatabase, including ferry routes. ${ }^{20,21}$ Each clinic was assigned a single wait time, either the wait for a caller with public insurance at clinics accepting public insurance or the wait for a caller with private insurance at clinics accepting only private insurance. For each $\mathrm{CBG}$, the average distance to and wait time at the five nearest therapy clinics and the five nearest therapy clinics accepting public insurance for each therapy discipline were determined, as using values for the single nearest clinic would reveal identifiable information about clinics. Given the small number of clinics, only distance to the rehabilitation medicine clinic nearest to the center point was determined for each CBG to avoid disclosure of individual clinic information.

Linear mixed models with robust variance and clinics nested in CBGs for each discipline were used to estimate mean differences in distance from the CBG center point to the five nearest clinics accepting public insurance and the five nearest clinics. Area-value maps (choropleths) of the mean distance and wait time for each CBG were created in order to visually compare access to rehabilitation services across geographic locations and insurance types.

Statistical significance was set at $P<.05$. ArcGIS Desktop 10.4 was used for geospatial analysis. Stata 12 software was used for data analysis. 


\section{Results}

Of the 427 clinics in the sample, 400 clinics were contacted and 287 indicated they could provide rehabilitation services for the simulated child with TBI, resulting in data from 195 physical therapy clinics (PTc), 109 occupational therapy clinics (OTc), 102 speech therapy clinics (STc), and 11 rehabilitation medicine clinics (Figure 1). Most PTc were single discipline, while OTc and STc were predominantly multidisciplinary. The majority of STc and rehabilitation medicine clinics were located in large metropolitan areas with most PTc and OTc located in small/non-metropolitan areas. (Table 1)

\section{Insurance Acceptance}

Overall, 147 (75.4\%) PTc, 78 (71.6\%) OTc, 71 (69.6\%) STc and 10 (90.9\%) rehabilitation medicine clinics accepted public insurance. Therapy clinics were $33-42 \%$ more likely to accept private versus public insurance while there was no significant difference in insurance plans accepted by rehabilitation medicine clinics. Multidisciplinary clinics were more likely than single discipline clinics to accept public insurance. Less than half of single discipline OTc and STc accepted public insurance. A higher proportion of clinics in small/nonmetropolitan areas accepted public insurance compared to those in large metropolitan areas. (Table 2)

\section{Wait Times for Rehabilitation Services}

When comparing the median wait time at clinics accepting public insurance versus only private insurance, there were statistically significant differences for PTc ( 7 versus 3 business days to next available appointment, $\mathrm{p}<.0001$ ) and STc (20 versus 5 business days, $\mathrm{p}=.0006$ ), and neared statistical significance for OTc ( 12.5 versus 5 business days, $\mathrm{p}=.0584)$.

Stratifying by urban status revealed different effects on wait time for the various disciplines. The wait at STc accepting public insurance in large metropolitan areas was 38.5 business days compared to only 5 business days at clinics accepting only private insurance ( $P=.0059)$. Large metropolitan OTc accepting public insurance had longer wait times than those accepting only private insurance (median 20 versus 5 business days, $\mathrm{p}=.0182$ ) while there was no significant difference for PTc in large metropolitan areas (4 versus 3 business days, $\mathrm{p}=.2239$ ). (Table 3 ) Rehabilitation medicine clinics had a median wait of 13 business days (interquartile range (IQR) 9-44, range 4-80), regardless of insurance type accepted.

When examining the distribution of difference in wait times within the $147 \mathrm{PTc}, 78$ OTc and 70 STc that accepted both insurance types, most clinics had no within-clinic insurance-based difference in wait time (median difference of 0 business days for all disciplines, IQR 0-1). However, the wait when the caller to these clinics had public insurance ranged up to 50 (PTc), 55 (OTc), and 115 (STc) business days longer than when the caller had private insurance.

\section{Multivariable analysis}

Adjusting for urban and multidisciplinary status, the wait until the next available appointment at clinics accepting public insurance was 59\% longer (count ratio (CR) 1.59, 95\% confidence interval (CI) 1.39-1.81) for PTc, $18 \%$ longer (CR 1.18, 95\% CI 1.07-1.30) 
for OTc and 107\% longer (CR 2.07, 95\% CI 1.87-2.30) for STc compared to clinics accepting only private insurance. Urban status remained statistically significant for OTc and STc and multidisciplinary status affected wait times for PTc and STc when adjusting for other covariates. (Table 4)

\section{Geospatial Analysis}

The average distance from the CBG center point to the five nearest clinics varied by discipline (PTc 11.0 miles (SD 13.5); OTc 15.0 miles (SD 17.8); STc 17.4 miles (SD 21.5), rehabilitation medicine 60.7 miles (SD 37.5)). The difference in mean distance from the CBG center point to the five nearest clinics and the mean distance to the five nearest clinics accepting public insurance also varied by discipline, with the greatest difference among STc (mean difference 6.2 miles (95\% CI 5.8, 6.9)). The visual representation of the average distance traveled and wait time for therapy services by CBG demonstrates geographic variation across the state (supplemental figures 1 and 2).

\section{Discussion}

In this study, callers posing as mothers of a simulated child with TBI covered by public insurance in Washington State were less likely to have their insurance accepted and had longer waits for therapy services than callers with private insurance. More than a quarter of therapy clinics sampled did not accept public insurance, a finding that corroborates previous work ${ }^{10}$ and suggests barriers to care following pediatric TBI in Washington State.

Median wait times at therapy clinics accepting public insurance were 4 to 15 business days longer than at clinics accepting only private insurance. This represents less disparity than found in other insurance-based studies of access to care..$^{5-7}$ These studies did not include analyses comparing access based on urban status. In this study, disparities in the proportion of therapy clinics accepting public insurance were more pronounced in large metropolitan areas. It is possible that the greater population density in large metropolitan areas makes it feasible for urban clinics to fill a practice while only accepting private insurance, reducing access options for those with public insurance.

The geospatial analysis revealed a number of findings. As might be expected, travel distances were longer for rehabilitation medicine access than for any of the therapy services since there were only 11 such clinics in the state. While the differences in travel times by insurance status for different services were relatively small, the effect of insurance type on wait times varied by region of the state. Federally qualified health centers and other clinics which provide care for children with public insurance should consider adding therapy services to their program to increase access to rehabilitation services.

Among the therapy disciplines, STc were least likely to accept public insurance and had the largest insurance-based differences in wait times. This is concerning, as a prior study found children 12 months after TBI had greater need for cognitive health services - such as those provided by STc - than physical or socioemotional services. ${ }^{2}$ This study's findings suggest the rehabilitation therapy discipline in greatest demand may also have the most striking insurance-based access disparities. 


\title{
Study limitations
}

As a single state study, these results cannot be generalized to other states, particularly states which did not expand Medicaid under the Affordable Care Act. The injury profile assessed by this study was limited to the chronic sequela of TBI; access patterns may differ for children with acute TBI, such as those recently discharged from hospitals or rehabilitation units. The sample may not include every clinic offering outpatient pediatric neurorehabilitation therapies. Because the analysis focused on differences by insurance type, the sampling method did not utilize the insurance plans' provider directories, which means avenues for accessing rehabilitation services may have been missed. Excluding clinics focusing on musculoskeletal impairments might have undercounted children's access to rehabilitation services. However, the study team felt a child with TBI would be best served by clinics specializing in pediatric or neurological rehabilitation. Multiple callers collected this data, though all were trained by one individual (L.T.). The analysis did not adjust for the number of providers at a clinic, clinic's popularity or reputation for quality services, or whether the clinic was hospital-based, all of which could affect the wait time for an appointment and likelihood of public insurance acceptance. Future studies should include details on clinic infrastructure, which will need to be obtained using a different methodology than used in this study. This analysis focused on wait times to initial evaluation and not ongoing treatments. While there may be different patterns of access for ongoing outpatient pediatric rehabilitation services, barriers to accessing initial evaluations - such as those described in this study - preclude opportunities for ongoing service delivery.

\section{Conclusions}

Further work is needed to characterize and mitigate factors influencing disparities in access to outpatient rehabilitation services for children with public insurance. It is not known whether the disparities found in this study were a result of intentional policies or implicit bias. Regardless, this study's findings serve as a call to action. Along with policy makers, rehabilitation professionals should implement changes to promote equitable access to rehabilitation care.

\section{Supplementary Material}

Refer to Web version on PubMed Central for supplementary material.

\section{Acknowledgement of Funding Support}

This work was supported by the Seattle Sports Concussion Research Collaborative; the Satterberg Foundation; the National Institute of Child Health and Human Development (grant number 5T32HD057822-05); and the National Center For Advancing Translational Sciences of the National Institutes of Health (grant number UL1TR000423). The funding agencies had no involvement in any phase of study conduct. The content is solely the responsibility of the authors and does not necessarily represent the official views of the National Institutes of Health.

Other Acknowledgements: We would like to thank Lauren Balsalmo, Marni Levy and Deana Rich for their assistance with data collection.

\section{Abbreviations}

\author{
CBG Census Block Group
}




$\begin{array}{ll}\text { CHIP } & \text { Children's Health Insurance Program } \\ \text { CI } & \text { Confidence Interval } \\ \text { CR } & \text { Count Ratio } \\ \text { IQR } & \text { Interquartile Range } \\ \text { OTc } & \text { Occupational Therapy Clinics } \\ \text { PTc } & \text { Physical Therapy Clinics } \\ \text { STc } & \text { Speech Therapy Clinics } \\ \text { TBI } & \text { Traumatic Brain Injury }\end{array}$

\section{References}

1. Faul, M., Xu, L., Wald, MM., Coronado, VG., National Center for Injury Prevention and Control. Traumatic brain injury in the United States: emergency department visits, hospitalizations, and deaths, 2002-2006. U.S. Dept. of Health and Human Services, Centers for Disease Control and Prevention, National Center for Injury Prevention and Control; Atlanta, GA: 2010. http:// www.cdc.gov/traumaticbraininjury/pdf/blue_book.pdf

2. Slomine BS, McCarthy ML, Ding R, et al. Health care utilization and needs after pediatric traumatic brain injury. Pediatrics. 2006; 117(4):e663-674. [PubMed: 16533894]

3. Greene NH, Kernic MA, Vavilala MS, Rivara FP. Variation in pediatric traumatic brain injury outcomes in the United States. Arch Phys Med Rehabil. 2014; 95(6):1148-1155. [PubMed: 24631594]

4. Skinner AC, Mayer ML. Effects of insurance status on children's access to specialty care: a systematic review of the literature. BMC Health Serv Res. 2007; 7:194. [PubMed: 18045482]

5. Bisgaier J, Rhodes KV. Auditing access to specialty care for children with public insurance. New Engl J Med. 2011; 364(24):2324-2333. [PubMed: 21675891]

6. Bisgaier J, Polsky D, Rhodes KV. Academic medical centers and equity in specialty care access for children. Arch Pediatr Adolesc Med. 2012; 166(4):304-310. [PubMed: 22147760]

7. Penn EB Jr. French A, Bhushan B, Schroeder JW Jr. Access to care for children with symptoms of sleep disordered breathing. nt J Pediatr Otorhinolaryngol. 2012; 76(11):1671-1673.

8. Chaudhry SB, Armbrecht ES, Shin Y, et al. Pediatric access to dermatologists: Medicaid versus private insurance. J Am Acad Dermatol. 2013; 68(5):738-748. [PubMed: 23474423]

9. Schiraldi M, Patil CG, Mukherjee D, et al. Effect of insurance and racial disparities on outcomes in traumatic brain injury. J Neurol Surg A Cent Eur Neurosurg. 2015; 76(3):224-232. [PubMed: 25798799]

10. Moore M, Jimenez N, Rowhani-Rahbar A, et al. Availability of Outpatient Rehabilitation Services for Children After Traumatic Brain Injury: Differences by Language and Insurance Status. Am J Phys Med Rehabil. 2016; 95(3):204-213. [PubMed: 26259055]

11. Kaiser Family Foundation. [March 16, 2016] State Health Facts: Health Insurance Coverage of Children 0-18. 2016. http://kff.org/other/state-indicator/children-0-18/?state=WA.

12. Medicaid and CHIP Payment and Access Commission. [October 17, 2016] Medicaid and CHIP Income Eligibility Levels as a Percentage of the FPL for Children and Pregnant Women by State, July 2016. 2016. https://www.macpac.gov/wp-content/uploads/2015/01/EXHIBIT-34.-Medicaidand-CHIP-Income-Eligibility-Levels-as-a-Percentage-of-the-FPL-for-Children-and-PregnantWomen-by-State-July-2016.pdf.

13. Medicaid and CHIP Payment and Access Commission. [October 17, 2016] Child Enrollment in CHIP and Medicaid by State, FY 2015. 2015. https://www.macpac.gov/wp-content/uploads/ 2015/01/EXHIBIT-31-Child-Enrollment-in-CHIP-Medicaid-by-state-FY-2015-1.pdf. 
14. Excel (Part of Microsoft Office Professional Edition) [computer program]. Microsoft; 2010.

15. Google. [January 11, 2016] Google Maps. https://www.google.com/maps/.

16. United States Department of Agriculture Economic Research Service. [July 14, 2014] Urban Influence Codes. 2013. http://ers.usda.gov/data-products/urban-influence-codes/ documentation.aspx.

17. Zou G. A modified poisson regression approach to prospective studies with binary data. Am J Epidemiol. 2004; 159(7):702-706. [PubMed: 15033648]

18. Schneider, A. [20 Jun, 2016] GPS Visualizer Address Locator. 2016. http:// www.gpsvisualizer.com/geocoder/.

19. Bing ${ }^{\text {TM }}$ Maps REST (Representational State Transfer) Services API (Application Programming Interface): Locations. Microsoft Corporation; 2016. https://msdn.microsoft.com/en-us/library/ ff701715.aspx. [20 Jun 2016]

20. TIGER/Line ${ }^{\circledR}$ Shapefiles and TIGER/Line ${ }^{\circledR}$ Files. US Department of Commerce; 2015. https:// www.census.gov/geo/maps-data/data/tiger-line.html. [20 Jun 2016]

21. Washington All Public Roads (WAPR). [23 May 2016] Washington State. 2014. http:// www.wsdot.wa.gov/mapsdata/geodatacatalog/. 


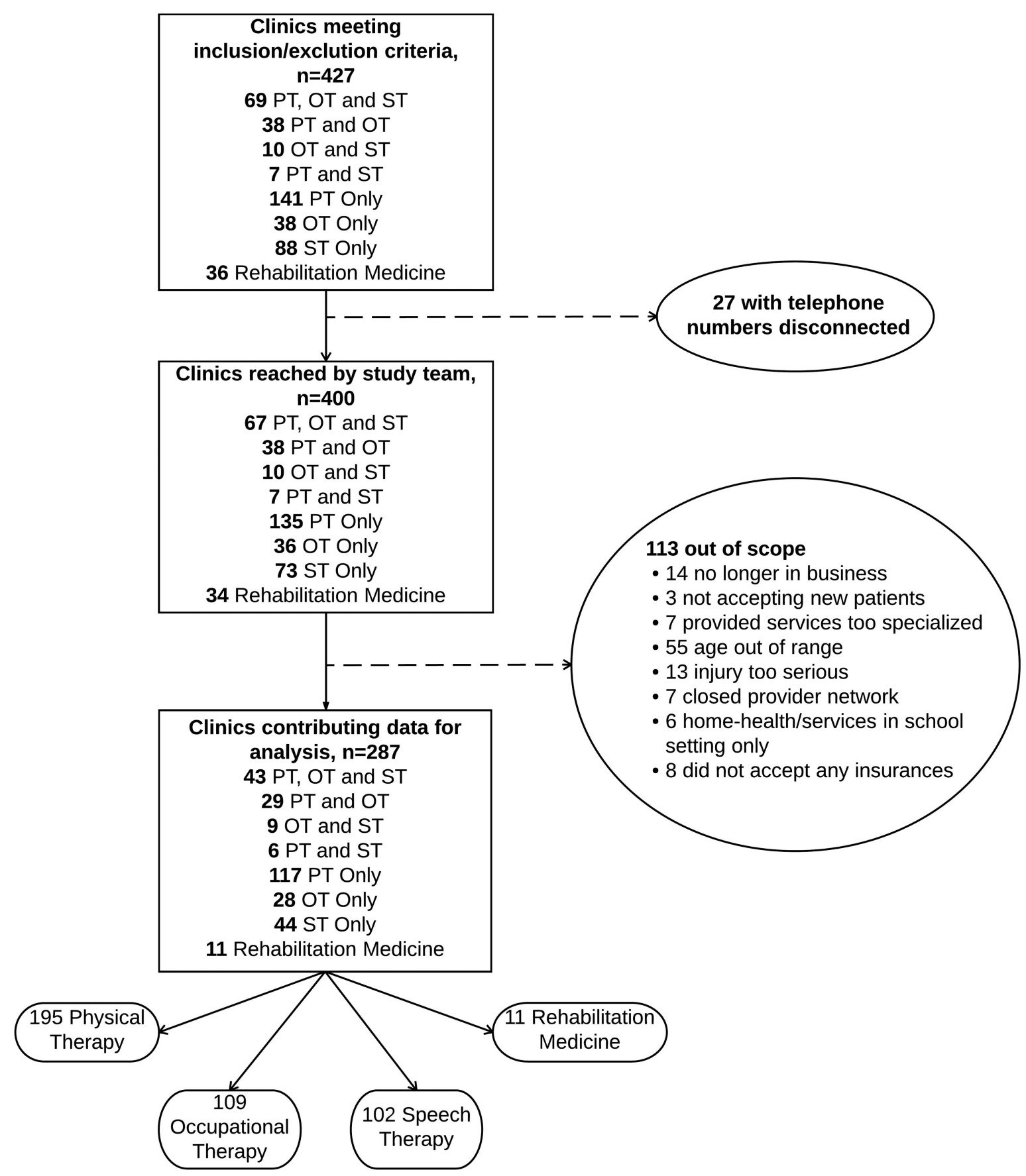

Figure 1.

Clinics included in study sample. Abbreviations: Physical Therapy (PT), Occupational Therapy (OT), Speech Therapy (ST) 


\section{Table 1}

Characteristics of outpatient rehabilitation clinics.

\begin{tabular}{|l|c|c|c|c|}
\hline & $\underline{\text { Rehabilitation Medicine Clinics }}$ & $\underline{\text { Physical Therapy Clinics }}$ & $\underline{\text { Occupational Therapy Clinics }}$ & $\underline{\text { Speech Therapy Clinics }}$ \\
\hline & $(\mathbf{N}=\mathbf{1 1})$ & $(\mathbf{N}=\mathbf{1 9 5})$ & $(\mathbf{N}=\mathbf{1 0 9})$ & $(\mathbf{N}=\mathbf{1 0 2})$ \\
\hline $\begin{array}{l}\text { Number of disciplines in } \\
\text { clinic }\end{array}$ & & & & \\
\hline Single discipline & $11(100.0)$ & $117(60.0)$ & $28(25.7)$ & $44(43.1)$ \\
\hline Multidisciplinary & $0(0.0)$ & $78(40.0)$ & $81(74.3)$ & $58(56.9)$ \\
\hline Urban location of clinic & & & & \\
\hline Large metro area & $6(54.6)$ & $80(41.0)$ & $49(45.0)$ & $54(52.9)$ \\
\hline Small metro/non-metro area & $5(45.4)$ & $115(59.0)$ & $60(55.0)$ & $48(47.1)$ \\
\hline
\end{tabular}

Note. Values presented as N (column \%). 
Table 2

Insurance plan acceptance at outpatient rehabilitation clinics, by insurance type

\begin{tabular}{|c|c|c|c|}
\hline & Clinic accepts private insurance & Clinic accepts public insurance & Relative Risk [95\% CI] \\
\hline & \multicolumn{2}{|c|}{$\mathbf{N}(\%)$} & \\
\hline \multicolumn{4}{|l|}{ All clinics } \\
\hline $\operatorname{PTc}(\mathrm{n}=195)$ & $195(100.0)$ & $147(75.4)$ & $1.33[1.22-1.44]$ \\
\hline OTc $(\mathrm{n}=109)$ & $109(100.0)$ & $78(71.6)$ & $1.40[1.24-1.57]$ \\
\hline $\operatorname{STc}(n=102)$ & $101(99.0)$ & $71(69.6)$ & $1.42[1.25-1.62]$ \\
\hline Rehab Med (n=11) & $11(100.0)$ & $10(90.9)$ & $1.10[0.90-1.34]$ \\
\hline \multicolumn{4}{|c|}{ Number of Disciplines in Clinic } \\
\hline \multicolumn{4}{|l|}{ Single Discipline } \\
\hline $\operatorname{PTc}(n=117)$ & $117(100.0)$ & $84(71.8)$ & $1.39[1.24-1.56]$ \\
\hline OTc $(n=28)$ & $28(100.0)$ & $12(42.9)$ & $2.33[1.51-3.61]$ \\
\hline $\operatorname{STc}(n=44)$ & $44(100.0)$ & $21(47.8)$ & $2.10[1.53-2.86]$ \\
\hline \multicolumn{4}{|l|}{ Multidisciplinary } \\
\hline PTc $(n=78)$ & $78(100.0)$ & $63(80.8)$ & $1.24[1.11-1.38]$ \\
\hline OTc $(n=81)$ & $81(100.0)$ & $68(84.0)$ & $1.23[1.11-1.36]$ \\
\hline $\mathrm{STc}(\mathrm{n}=58)$ & $57(98.3)$ & $49(84.5)$ & $1.14[1.02-1.27]$ \\
\hline \multicolumn{4}{|c|}{ Urban Location of Clinic } \\
\hline \multicolumn{4}{|l|}{ Large metro area } \\
\hline $\operatorname{PTc}(n=80)$ & $80(100.0)$ & $53(66.3)$ & $1.51[1.29-1.77]$ \\
\hline OTc $(n=49)$ & $49(100.0)$ & $26(53.1)$ & $1.88[1.44-2.46]$ \\
\hline $\operatorname{STc}(n=54)$ & $54(100.0)$ & $28(51.9)$ & $1.93[1.49-2.50]$ \\
\hline Rehab Med (n=6) & $6(100.0)$ & $5(83.3)$ & $1.20[0.81-1.78]$ \\
\hline \multicolumn{4}{|c|}{ Small metro/Non-metro area } \\
\hline $\operatorname{PTc}(n=115)$ & $115(100.0)$ & $94(81.7)$ & $1.22[1.12-1.33]$ \\
\hline OTc $(n=60)$ & $60(100.0)$ & $52(86.7)$ & 1.15 [1.04-1.28] \\
\hline $\operatorname{STc}(n=48)$ & $47(97.9)$ & $43(89.6)$ & $1.09[0.98-1.22]$ \\
\hline Rehab Med (n=5) & $5(100.0)$ & $5(100.0)$ & -- \\
\hline
\end{tabular}

Note: Relative risk in this generalized linear model compares proportion of clinics offering an appointment when the caller had private insurance versus public insurance. All rehabilitation medicine clinics were single disciplinary.

Abbreviations: CI, confidence interval; PTc, physical therapy clinic; OTc, occupational therapy clinic; STc, speech therapy clinic; Rehab Med, rehabilitation medicine clinic 


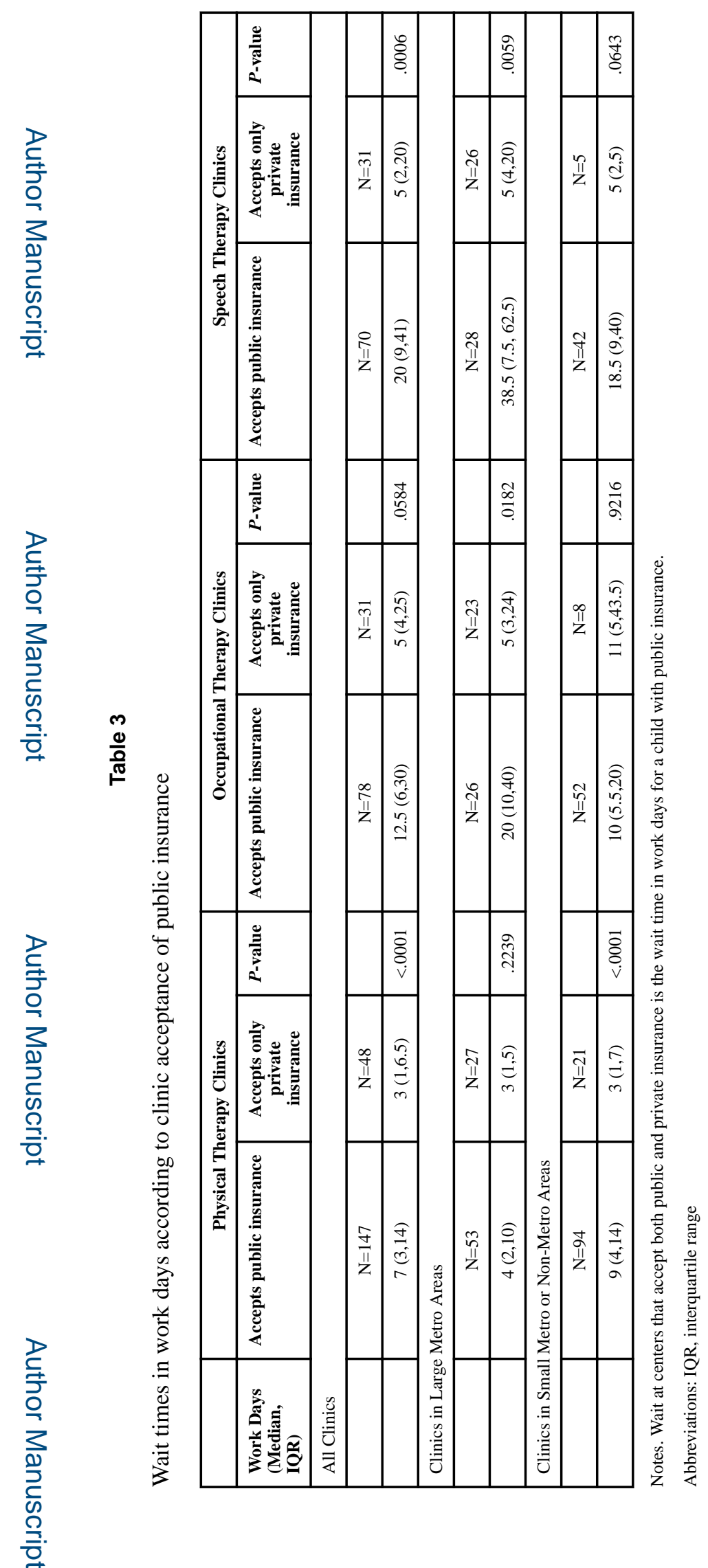

Arch Phys Med Rehabil. Author manuscript; available in PMC 2018 September 01. 


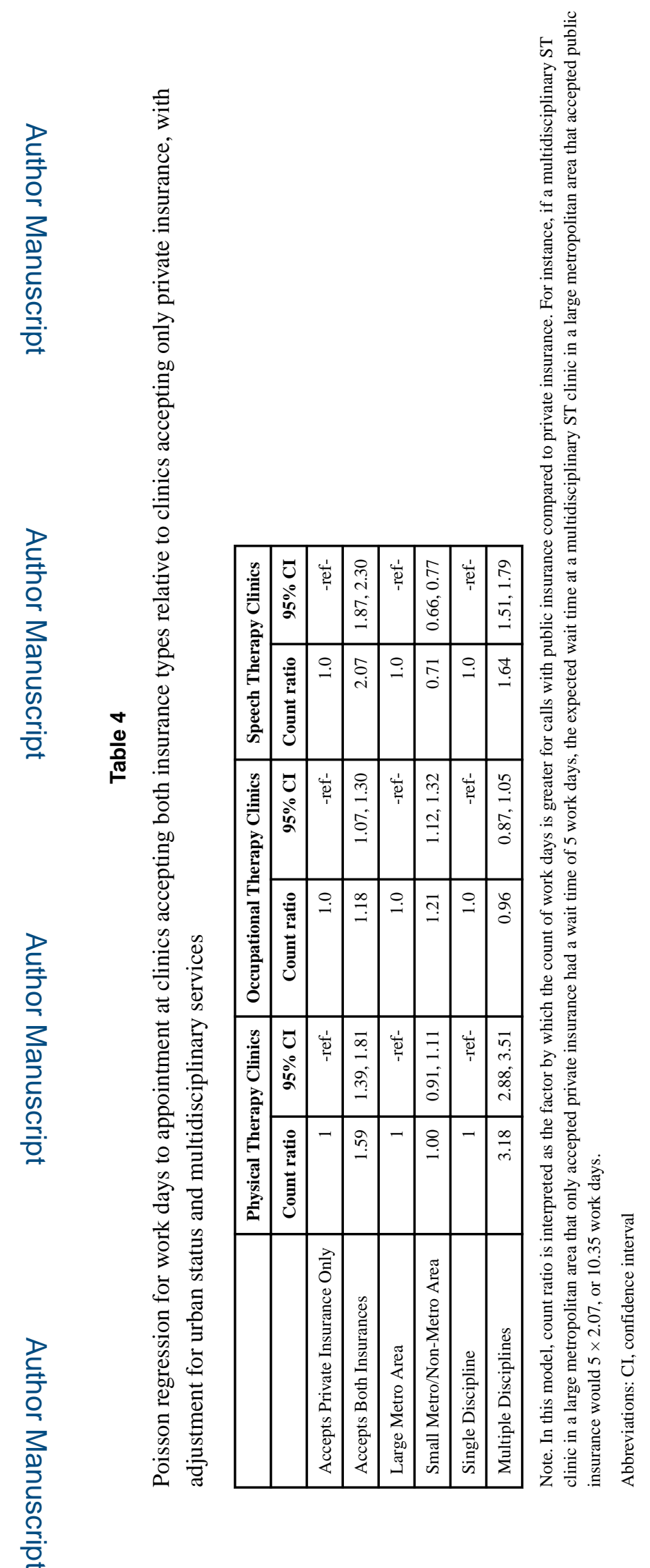

Arch Phys Med Rehabil. Author manuscript; available in PMC 2018 September 01. 